\title{
Search for upward-going showers with the Fluorescence Detector of the Pierre Auger Observatory
}

\author{
Massimo Mastrodicasa ${ }^{a, *}$ on behalf of the Pierre Auger $^{b}$ Collaboration \\ (a complete list of authors can be found at the end of the proceedings) \\ ${ }^{a}$ Università dell'Aquila, Dipartimento di Scienze Fisiche e Chimiche, L'Aquila, Italy and INFN Laboratori \\ Nazionali del Gran Sasso, Assergi (L'Aquila), Italy \\ ${ }^{b}$ Observatorio Pierre Auger, Av. San Martín Norte 304, 5613 Malargüe, Argentina \\ E-mail: spokespersons@auger.org
}

Given its operation time and wide field of view, the Fluorescence Detector (FD) of the Pierre Auger Observatory is sufficiently sensitive to detect upward-going events when used in monocular mode. Upward-going air showers are a possible interpretation of the recent events reported by the ANITA Collaboration in the energy range above $10^{17} \mathrm{eV}$. The Pierre Auger FD data can be used to support or constrain this interpretation. If confirmed, it would require either new phenomena or significant modifications to the standard model of particle physics.

To prepare this search, a set of quality selection criteria was defined by using $10 \%$ of the available FD data from 14 years of operation. This subset was mainly used to clean the data from improperly labelled laser events that had been used to monitor the quality of the atmosphere. The potential background for this search consists of cosmic-ray induced air showers with specific geometric configurations which, in a monocular reconstruction, can be reconstructed erroneously as upwardgoing events. To distinguish candidates from these false positives, to calculate the exposure, and to estimate the expected background, dedicated simulations for signal (upward-going events) and background (downward-going events) have been performed. The detector exposure is large enough to strongly constrain the interpretation of ANITA anomalous events. Preliminary results of the analysis after unblinding the data set are presented.

$3^{\text {th }}$ International Cosmic Ray Conference (ICRC 2021)

July 12th - 23rd, 2021

Online - Berlin, Germany

\footnotetext{
${ }^{*}$ Presenter
} 


\section{Introduction}

The ANITA Collaboration has recently reported two events that are consistent with the interpretation of upward-going air showers. The two events were detected during the first and the third flights of ANITA with an elevation angle of $27.4 \pm 0.3^{\circ}$ [1] and $35.0 \pm 0.3^{\circ}$ [2], respectively, and energies above $\sim 0.2 \mathrm{EeV} \mathrm{[3].}$

The energies and the elevation angles of these two events appear challenging to reconcile with the predictions of the standard model of particle physics [2], so a confirmation or a constraint from a different experiment would be of particular interest.

The Pierre Auger Collaboration has performed a generic search for cosmic-ray-like upwardgoing air showers with the Fluorescence Detector (FD) of the Pierre Auger Observatory which consists of multiple telescopes that collect the fluorescence light emitted by Nitrogen as the shower front crosses the atmosphere [4]. The sensitivity of the FD to upward-going air showers has been studied with dedicated simulations of upward-going events distributed in the energy and zenith angle regions of interest. The potential background from mis-reconstructed downward-going showers has been estimated with high statistics simulations of downward-going events to which a monocular event reconstruction was applied. It was found that specific geometric configurations could be incorrectly reconstructed as upward-going events. Moreover, a sample of $10 \%$ of the available FD data (burn data sample) has been used to develop a set of cuts to reject laser events. These cuts together with those defined in simulations to minimize the confusion with downward-going air showers were finally applied to the burn data samples and the simulations to quantify the signal efficiency as well as the background expectation in the blinded data sample.

\section{Signal simulation}

Upward-going air showers can be initiated by particles emerging from the Earth and interacting or decaying in the atmosphere at a certain height or within the rock just below the Earth's crust. For large zenith angles $\left(\theta \gg 90^{\circ}\right)$, they are unlikely to exhibit a signal in the Surface Detector (SD), but they can be detected with the FD. The impact point of the particle trajectory on the ground will be referred as the "exit point". To calculate the FD exposure, upward-going air showers have been simulated with CONEX [5] and reconstructed within the Offline Framework [6]. Simulations indicate that the FD has neglible efficiency for shower energies below $10^{16.5} \mathrm{eV}$ and this study has been performed considering showers in the restricted energy interval $\log _{10}\left(E_{\mathrm{cal}} / \mathrm{eV}\right) \in$ $[16.5,18.5]$, within which the ANITA events comfortably fall. We note that showers of higher energy could of course be also potentially observed. The geometry of an upward-going shower

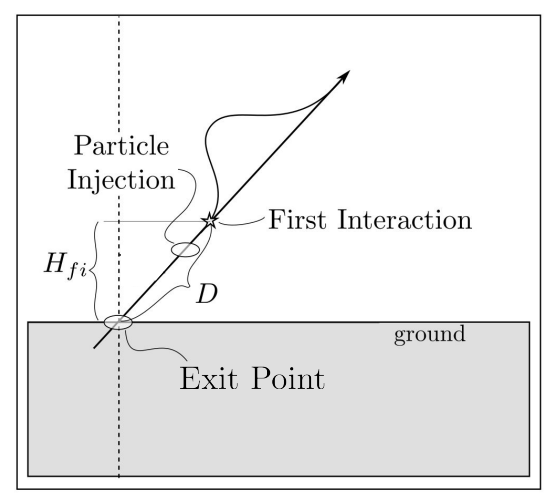

Figure 1: Schematic view of the generation geometry of an upward-going shower. $D$ is the distance along the shower axis of the point of first interaction of the primary particle from the exit point and $H_{\mathrm{fi}}$ is the height of the point of first interaction with respect to the ground. 
is illustrated in Figure 1. Simulations have been made according to an energy distribution of $E^{-1}$ and assuming an isotropic distribution of events. Only elevation angles in the range $[20,90]^{\circ}$ (zenith angle $(\theta)$ range $[110,180]^{\circ}$ ) are considered. Finally the height of the point of first interaction of the primary particle, $H_{\mathrm{fi}}$ is of relevance for establishing the energy of the ANITA events. Moreover, showers that start at high altitudes are naturally less likely to be triggered because they tend to be further away. The simulated showers have been generated in the region $[0,9] \mathrm{km}$ above the ground altitude of the Observatory $(\sim 1400 \mathrm{~m}$ a.s.l) with a uniform distribution. The exit points have been sampled in a square area of $100 \times 100 \mathrm{~km}^{2}$ centered at the SD station closest to the center of the SD array. This area extends up to $\sim 20 \mathrm{~km}$ behind each FD site, allowing to simulate also particles whose exit point is located behind the field of view of a telescope. The simulation is performed aproximating the start of the shower with a single proton, and it can be adapted to fit other scenarios. Sibyll 2.3c [7, 8] and UrQMD 1.3 [9] have been used as hadronic models at high and low energies, respectively.

To take into account all the FD configurations and their time variability during the 14 years of operation, a time dependent detector simulation has been performed [10].

\section{Exposure calculation}

The FD exposure, $\varepsilon\left(E_{\text {cal }}\right)$, is calculated as:

$$
\varepsilon\left(E_{\mathrm{cal}}\right)=A\left(E_{\mathrm{cal}}\right) \cdot \Delta T
$$

where $A$ is the Monte Carlo time-averaged FD aperture, $\Delta T$ is the observation time (i.e. the 14 years of operation of the FD) and $E_{\mathrm{cal}}$ is the energy deposited in the atmosphere (calorimetric). The aperture of the FD is defined as:

$$
A\left(E_{\mathrm{cal}}\right)=\int_{\Omega} S_{\mathrm{eff}}\left(E_{\mathrm{cal}}, \theta\right) \cos \theta d \Omega
$$

where $\Omega$ is the solid angle, $\theta$ is the zenith angle and $S_{\text {eff }}\left(E_{\text {cal }}\right)$ is the effective area, that is defined as:

$$
S_{\mathrm{eff}}\left(E_{\mathrm{cal}}, \theta\right)=\int_{S_{\mathrm{gen}}} \eta\left(E_{\mathrm{cal}}, \theta\right) \cdot d S
$$

Here $S_{\text {gen }}$ is the surface area on the ground plane over which exit points have been generated, a square of $100 \times 100 \mathrm{~km}^{2}$, and $\eta\left(E_{\mathrm{cal}}, \theta\right)$ is the Monte Carlo time-averaged detection efficiency at a specific energy and zenith angle. It is calculated as the ratio of the number of events that pass the selection criteria over the total number of generated events:

$$
\eta\left(E_{\text {cal }}, \theta\right)=\frac{n_{\text {selected }}\left(E_{\mathrm{cal}}, \theta\right)}{n_{\text {generated }}\left(E_{\mathrm{cal}}, \theta\right)} .
$$

Since the on-time fraction of the telescopes has been included in the signal simulation, it is automatically taken into account when $\eta(E, \theta)$ is calculated.

By inserting $S_{\text {eff }}$ from Eq. 3 into Eq. 2, the FD aperture becomes:

$$
A\left(E_{\mathrm{cal}}\right)=S_{\mathrm{gen}} \cdot \int_{\theta} \eta\left(E_{\mathrm{cal}}, \theta\right) \cos \theta d \cos \theta \cdot \int_{\phi} d \phi
$$


where the zenith angle range is $\theta \in[110,180]^{\circ}$ and the azimuth angle range is $\phi \in[0,360]^{\circ}$. Following the same approach as [10], the FD exposure at a specific energy is then given by:

$$
\varepsilon\left(E_{\mathrm{cal}}\right) \simeq 2 \pi \cdot S_{\mathrm{gen}} \cdot \Delta T \cdot \sum_{i} \eta\left(E_{\mathrm{cal}}, \cos \theta_{i}\right) \cdot \cos \theta_{i} \cdot \Delta \cos \theta_{i}
$$

Fixing the height of the point of first interaction, $H_{f i}$, Equation 6 can be used to calculate a differential exposure in $H_{f i}$. It is simply:

$$
\frac{d \varepsilon}{d H_{\mathrm{fi}}}\left(E_{\mathrm{cal}}, H_{\mathrm{fi}}\right) \simeq 2 \pi \cdot S_{\mathrm{gen}} \cdot \Delta T \cdot \sum_{i} \eta\left(E_{\mathrm{cal}}, \cos \theta_{i}, H_{\mathrm{fi}}\right) \cdot \frac{1}{\Delta H_{\mathrm{fi}}} \cdot \cos \theta_{i} \cdot \Delta \cos \theta_{i} .
$$

\section{Background simulation}

In this analysis FD data from a single location is typically the only data used for the reconstruction (monocular reconstruction). No SD data is required as upward-going showers cannot trigger the SD. Without this information downward-going events with specific geometries can be incorrectly reconstructed as upward-going events and vice-versa. An example is shown in Figure 2. The signal coming from point $\mathrm{P}_{1}$ arrives before that coming from point $\mathrm{P}_{2}$ because the impact point is behind the telescope mimicking the behaviour of an upward-going event. To study background events, a dedicated and extensive simulation of downward-going events has been performed. The FD is a volumetric detector, so the cores have been sampled in a sphere of radius $90 \mathrm{~km}$ around the FD rather than on a surface. Downward-going events have been simulated with an energy $\log _{10}(E / \mathrm{eV}) \in[17,20]$ and a zenith angle $\theta \in[0,90]^{\circ}$. Helium, nitrogen and iron nuclei, together with protons, have been considered as primary particles. The hadronic models at high and low energies used for the background simulations are the same as for the signal simulations. The background simulations have been used to study the topology of the background events and define selection criteria to discriminate between signal events and false positives induced by a small fraction of regular cosmic ray showers $(<0.1 \%)$.

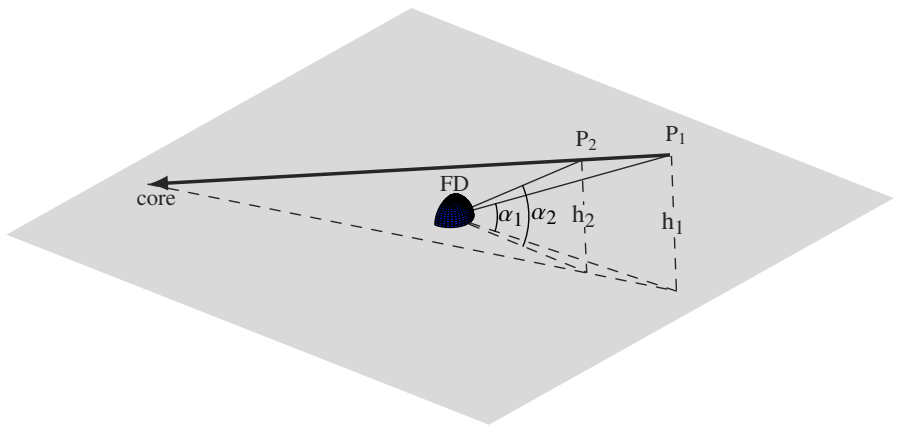

Figure 2: Schematic view of the geometry of a downward-going event that is reconstructed as an upward-going event. For the two points $\mathrm{P}_{1}$ and $\mathrm{P}_{2}$ on the shower axis $\alpha_{1}<\alpha_{2}$ and $\mathrm{h}_{1}>\mathrm{h}_{2}$. The signal from $\mathrm{P}_{1}$ reaches the FD before the signal from $\mathrm{P}_{2}$ and the event can be reconstructed as an upward-going event.

\section{Data cleaning and event selection criteria}

A set of selection cuts to preserve the quality of both the reconstruction and the atmospheric conditions has been defined and tested on signal and background simulations to select genuine upward-going reconstructed events. Of particular relevance is the identification of laser shots in the data sample. The FD requires continuous monitoring of the atmosphere and millions of laser 


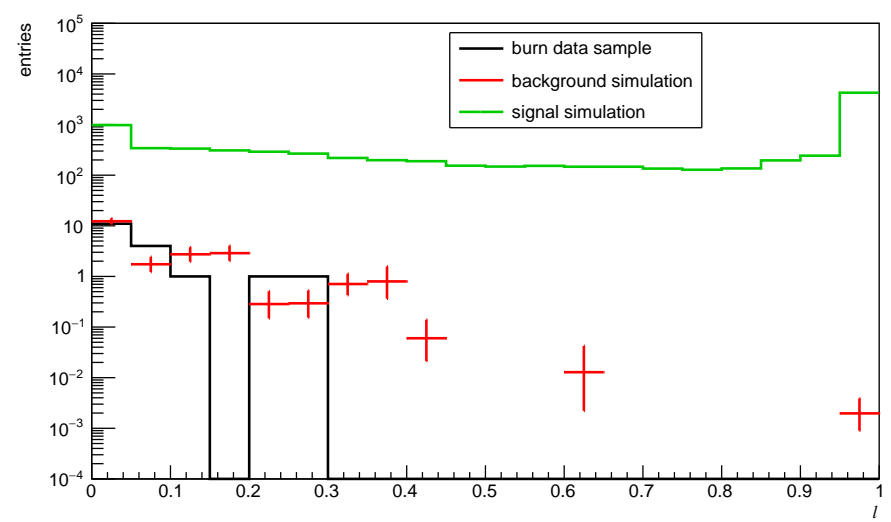

Figure 3: Distribution of $l$ for the burn data sample, the background and the signal simulations with all selection criteria applied. The background simulation has been weighted to the burn data sample and the Cosmic Ray (CR) energy spectrum [11]. It includes the events in the simulated energy range $\log _{10}(E / \mathrm{eV}) \in[17,20]$. The signal simulation has not been weighted and includes the events with a simulated calorimetric energy in the range $\log _{10}\left(E_{\text {cal }} / \mathrm{eV}\right) \in[16.5,18.5]$.

shots are being fired from different positions during data acquisition for this purpose [4]. These are upward-going events and constitute a background for this search. Laser shots are usually recorded and stored so that they can be easily removed while performing the analysis. However, in case they are not properly labelled, they can produce false positives. To study and properly identify such events, a sample made of the $10 \%$ of the available FD data (burn data sample) has been used. This provided the input to define a set of selection cuts to be applied to the event time windows and exit point position at ground in which these laser events are expected. By following this procedure, the burn data sample has been cleaned from all laser events.

To discriminate signal from background events, a dedicated selection cut has been studied. Each event of both signal simulation and background simulation, as well as of the FD data, is reconstructed using the Profile Constrained Geometry Fit Reconstruction (PCGF) [12] that forces the depth profile to match the approximately universal characteristics of air showers induced by most primaries with a well defined shower maximum of known width in matter depth $\left(\sim 100 \mathrm{~g} \mathrm{~cm}^{-2}\right)$. The PCGF reconstruction is run in two distinct modes, downward and upward modes. The (upward) downward mode searches for the maximum value of the likelihood in the zenith angle region $\left(\theta \in[90,180]^{\circ}\right) \theta \in[0,90]^{\circ}$. This results in two independent values of the maximum likelihood, $L_{\text {down }}$ and $L_{\text {up }}$ respectively from the downward and upward modes. By comparing $L_{\text {down }}$ with $L_{\text {up }}$, it is possible to discriminate between events that are more likely to be downward-going ( $L_{\text {down }}>L_{\text {up }}$ ) and vice-versa. The variable $-2 \log \left(L_{\text {down }} / \max \left(L_{\text {up }}, L_{\text {down }}\right)\right)$ can be used to discriminate between signal events and background events. The higher the probability for an event to be downward-going, the closer to zero it becomes. To have a quantity that is defined between 0 and 1 it is convenient to re-define it as:

$$
l=\frac{\arctan \left(-2 \log \left(L_{\mathrm{down}} / \max \left(L_{\mathrm{up}}, L_{\mathrm{down}}\right)\right) / 50\right)}{\pi / 2}
$$

According to Eq. 8, upward-going-like events have an $l$ close to 1, while downward-going-like events have an $l$ close to 0 . 


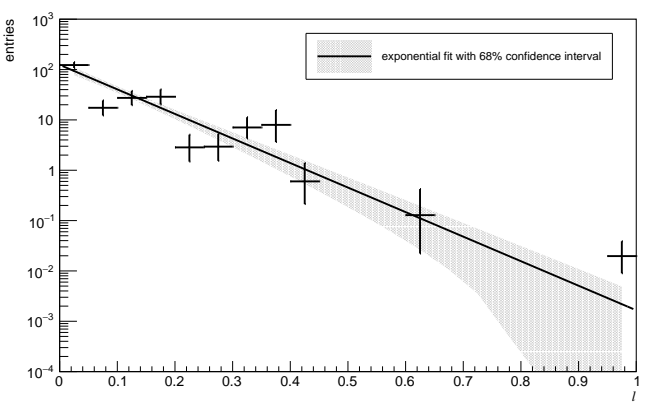

(a) Background distribution

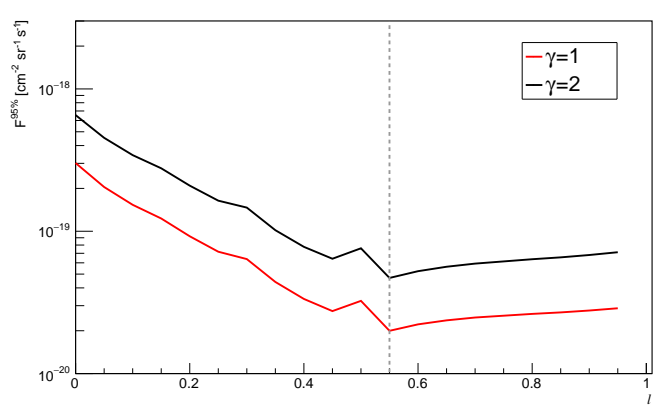

(b) Upper limit minimization

Figure 4: (a) Exponential fit performed via the weighted log likelihood method on the $l$-distribution for the background simulation weighted according to the CR energy spectrum. (b) Integral upper limit as a function of $l$ for $E_{\mathrm{cal}}>10^{17.5} \mathrm{eV}$ using this background fit and power-law indices $\gamma=1$ and $\gamma=2$ for the energy spectrum in the calculation of the average exposure. The integral upper limit has been calculated considering the background events in the whole energy range of simulation $\left(\log _{10}(E / e V) \in[17,20]\right)$. The minimum value of the integral upper limit has been found at $l=0.55$ (dashed grey line).

To set a cut value on $l$ and select events that are more likely upward-going, the distribution of this variable has been studied for signal simulations, background simulations and for the burn data sample (Fig. 3). The cut value has been chosen by making an assumption about the extrapolation of the background distribution and performing a scan on $l$ to find the value that minimizes the integral upper limit that could be set if no candidate events are observed after unblinding the data. The integral upper limit has been calculated as:

$$
F^{95 \%}\left(E_{\text {cal }}>E_{0}\right)=\frac{N^{95 \%}}{\langle\varepsilon\rangle}
$$

where $N^{95 \%}$ is the Rolke limit [13] on the number of upward-going events under the assumption that the number of observed events is equal to the number of background events expected from background and $\langle\varepsilon\rangle$ is the $E_{\text {cal }}^{-\gamma}$ weighted average exposure for $E_{\text {cal }}>E_{0}$, which is calculated as:

$$
\langle\varepsilon\rangle=\frac{\int_{E_{\mathrm{cal}}>E_{0}} E_{\mathrm{cal}}^{-\gamma} \varepsilon\left(E_{\mathrm{cal}}\right) d E_{\mathrm{cal}}}{\int_{E_{\mathrm{cal}}>E_{0}} E_{\mathrm{cal}}^{-\gamma} d E_{\mathrm{cal}}}
$$

where $E_{\text {cal }}$ is the simulated calorimetric energy and the calculation of $\varepsilon\left(E_{\text {cal }}\right)$ refers to a flat generation in $H_{\mathrm{fi}}$ and an isotropic emergence.

To calculate the number of events expected from background, the distribution for background simulation (c.f. Fig. 4 (a)) has been parameterized using different functions. For each of them, the value of $l$ yielding the minimum value of the integral upper limit has been searched for. The distribution of the background simulation fitted with an exponential function via the weighted $\log$ likelihood method is shown in Figure 4 (a). The resulting integral upper limit with $E_{0}=10^{17.5} \mathrm{eV}$ as a function of $l$ obtained using the result of the fit to estimate the number of events expected from background is shown in Figure 4 (b). The uncertainty coming from the fit is taken into account in the calculation of the Rolke limit. The minimum value of the integral upper limit has been found 
at $l=0.55$. This is the cut value that has been chosen as discrimination value between candidate events and background events. Using this cut value, the expected number of background events from the fit with the exponential function is:

$$
n_{\mathrm{bkg}}=0.45 \pm 0.18
$$

Different parameterizations of the background distribution, such as $\exp \left(a x^{2}+b x+c\right)$, resulted in slightly different cut values on $l$ but yielded the same upper limits within about $10 \%$. This uncertainty is included in the number $n_{\text {bkg }}$ of Eq. 11. After the unblinding of the data the integral upper limit has been calculated according to Eq. 9 using the number of events observed in the full data sample and Eq. 11 as expected number of background events.

\section{Results}

After performing the unblinding of the data, $n_{\mathrm{obs}}=1$ event has been observed to pass all the selection criteria in the full data sample, a number that is consistent with the expected number of background events. The integral upper limit obtained with this number of observed events for two different values of $\gamma(\gamma=1$ and $\gamma=2)$ is:

$$
\begin{aligned}
& F_{\gamma=1}^{95 \%}\left(E_{\mathrm{cal}}>10^{17.5} \mathrm{eV}\right)=3.6 \cdot 10^{-20} \mathrm{~cm}^{-2} \mathrm{sr}^{-1} \mathrm{~s}^{-1} \\
& F_{\gamma=2}^{95 \%}\left(E_{\mathrm{cal}}>10^{17.5} \mathrm{eV}\right)=8.5 \cdot 10^{-20} \mathrm{~cm}^{-2} \mathrm{sr}^{-1} \mathrm{~s}^{-1}
\end{aligned}
$$

To make this result applicable to different scenarios, differential tables of exposure are provided. The preliminary results for the differential exposure obtained according to Eq. 7 as a function of the height of first interaction and the simulated calorimetric energy for a flat generation in the height of first interaction and an isotropic emergence is shown in Figure 5. These differential tables can be used to set limits for different physical scenarios, such as the one that predicts upward-going showers initiated by $\tau$-leptons that are produced in the interaction of $\tau$-neutrinos with the Earth [14].

\section{Conclusions}

A search for upward-going showers has been performed with the FD of the Pierre Auger Observatory. The available data from 14 years of operation have been analyzed and $n_{\text {obs }}=1$ event has been found. The expected number of background events was $n_{\mathrm{bkg}}=0.45 \pm 0.18$, that resulted in an integral upper limit of $F_{\gamma=1}^{95 \%}\left(E_{\mathrm{cal}}>10^{17.5} \mathrm{eV}\right)=3.6 \cdot 10^{-20} \mathrm{~cm}^{-2} \mathrm{sr}^{-1} \mathrm{~s}^{-1}$ for the case of a $E_{\text {cal }}^{-1}$ weighted average exposure and $F_{\gamma=2}^{95 \%}\left(E_{\mathrm{cal}}>10^{17.5} \mathrm{eV}\right)=8.5 \cdot 10^{-20} \mathrm{~cm}^{-2} \mathrm{sr}^{-1} \mathrm{~s}^{-1}$ for the case of a $E_{\text {cal }}^{-2}$ weighted average exposure. Differential tables of exposure have been provided. These tables make the result of this search applicable to different physical scenarios. To compare these results with the ANITA observations a similar estimation of its exposure to upward-going showers as a function of energy and altitude would be needed. Such a comparison is planned to be addressed as a following step. 


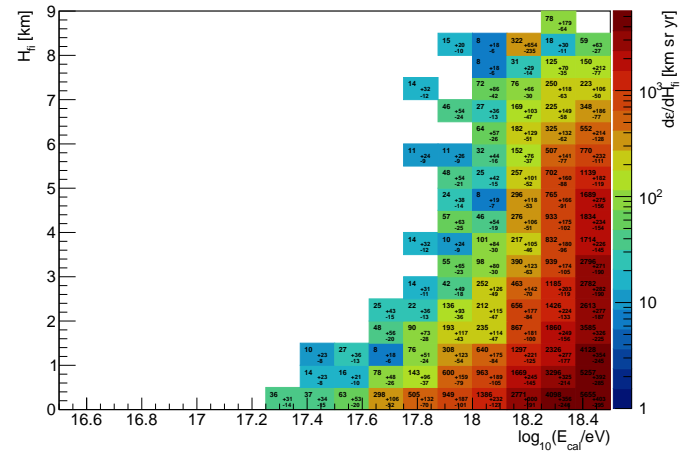

(a) $\theta \in[110,180]^{\circ}$

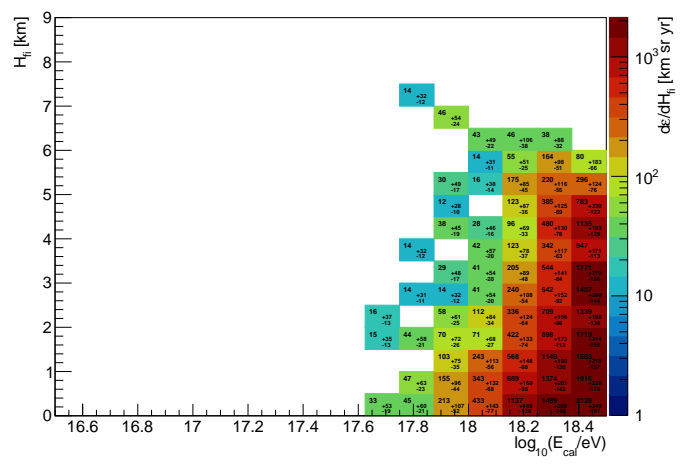

(c) $\theta \in[124.2,141.3]^{\circ}$

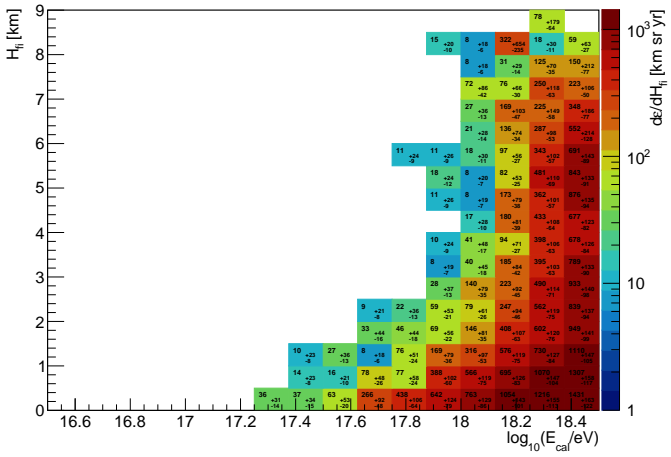

(b) $\theta \in[110,124.2]^{\circ}$

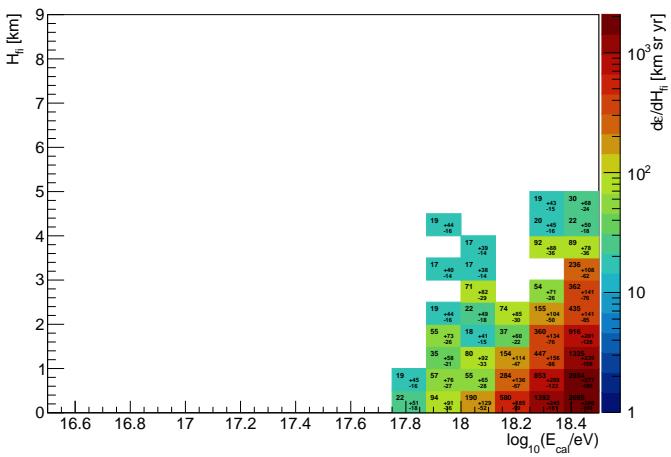

(d) $\theta \in[141.3,180]^{\circ}$

Figure 5: Preliminary double differential exposure obtained according to Eq. 7 as a function of $H_{\mathrm{fi}}$ and $E_{\text {cal }}$ and for different ranges of zenith angles. The exposure calculation refers to a flat generation in $H_{\mathrm{fi}}$ and an isotropic emergence.

\section{References}

[1] P. W. Gorham et al. [ANITA Coll.], Phys. Rev. Lett. 117 (2016) 071101, [1603. 05218].

[2] P. W. Gorham et al. [ANITA Coll.], Phys. Rev. Lett. 121 (2018) 161102, [1803. 05088].

[3] A. Romero-Wolf, S. A. Wissel, H. Schoorlemmer, W. R. Carvalho, J. Alvarez-Muñiz, E. Zas, P. Allison, O. Banerjee, L. Batten and J. J. Beatty, et al., Phys. Rev. D99 (2019) 063011, [1811.07261]

[4] A. Aab et al. [Pierre Auger Coll.], Nucl. Instrum. Meth. A 798 (2015) 172-213, [1502.01323].

[5] T. Bergmann, R. Engel, D. Heck, N. N. Kalmykov, S. Ostapchenko, T. Pierog, T. Thouw and K. Werner, Astropart. Phys. 26 (2007) 420-432, [astro-ph/0606564].

[6] S. Argiro, S. L. C. Barroso, J. Gonzalez, L. Nellen, T. C. Paul, T. A. Porter, L. Prado, Jr., M. Roth, R. Ulrich and D. Veberic, Nucl. Instrum. Meth. A 580 (2007) 1485-1496, [0707. 1652].

[7] F. Riehn, H. P. Dembinski, R. Engel, A. Fedynitch, T. K. Gaisser and T. Stanev, Proc. 35th Int. Cosmic Ray Conf., Busan, Korea (2017), PoS(ICRC2017)301, [1709.07227].

[8] E. J. Ahn, R. Engel, T. K. Gaisser, P. Lipari and T. Stanev, Phys. Rev. D 80 (2009) 094003, [0906. 4113].

[9] S. A. Bass, M. Belkacem, M. Bleicher, M. Brandstetter, L. Bravina, C. Ernst, L. Gerland, M. Hofmann,

S. Hofmann and J. Konopka, et al. Prog. Part. Nucl. Phys. 41 (1998) 255-369, [nucl-th/9803035].

[10] P. Abreu et al. [Pierre Auger Coll.], Astropart. Phys. 34 (2011) 368-381, [1010.6162].

[11] A. Aab et al. [Pierre Auger Coll.] Phys. Rev. Lett. 125 (2020) 121106, [2008. 06488].

[12] V. Novotny [for the Pierre Auger Coll.], Proc. 36th Int. Cosmic Ray Conf., Madison, WI, U.S.A. (2019), PoS(ICRC2019)374, [1909.09073].

[13] W. A. Rolke, A. M. Lopez and J. Conrad, Nucl. Instrum. Meth. A 551 (2005) 493-503, [physics/0403059].

[14] I. Caracas [for the Pierre Auger Coll.], these proceedings. 


\section{The Pierre Auger Collaboration}

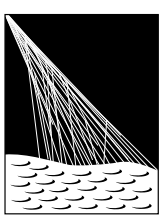

PIERRE

AUSGERVATORY

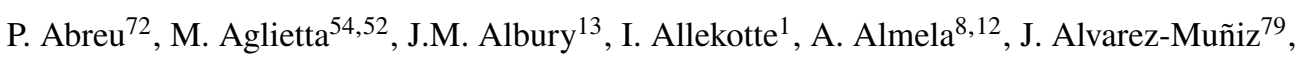
R. Alves Batista ${ }^{80}$, G.A. Anastasi ${ }^{63,52}$, L. Anchordoqui ${ }^{87}$, B. Andrada ${ }^{8}$, S. Andringa ${ }^{72}$, C. $\mathrm{Aramo}^{50}$, P.R. Araújo Ferreira ${ }^{42}$, J. C. Arteaga Velázquez ${ }^{67}$, H. Asorey $^{8}$, P. Assis ${ }^{72}$, G. Avila ${ }^{11}$, A.M. Badescu ${ }^{75}$, A. Bakalova ${ }^{32}$, A. Balaceanu ${ }^{73}$, F. Barbato ${ }^{45,46}$, R.J. Barreira Luz $^{72}$, K.H. Becker ${ }^{38}$, J.A. Bellido ${ }^{13,69}$, C. Berat ${ }^{36}$, M.E. Bertaina ${ }^{63,52}$, X. Bertou ${ }^{1}$, P.L. Biermann ${ }^{b}$, V. Binet ${ }^{6}$, K. Bismark ${ }^{39,8}$, T. Bister ${ }^{42}$, J. Biteau ${ }^{37}$, J. Blazek ${ }^{32}$, C. Bleve ${ }^{36}$, M. Boháčová ${ }^{32}$, D. Boncioli ${ }^{57,46}$, C. Bonifazi ${ }^{9,26}$, L. Bonneau Arbeletche ${ }^{21}$, N. Borodai ${ }^{70}$, A.M. Botti ${ }^{8}$, J. Brack ${ }^{d}$, T. Bretz ${ }^{42}$, P.G. Brichetto Orchera ${ }^{8}$, F.L. Briechle ${ }^{42}$, P. Buchholz ${ }^{44}$, A. Bueno ${ }^{78}$, S. Buitink ${ }^{15}$, M. Buscemi ${ }^{47}$, M. Büsken ${ }^{39,8}$, K.S. Caballero-Mora ${ }^{66}$, L. Caccianiga ${ }^{59,49}$, F. Canfora ${ }^{80,81}$, I. Caracas ${ }^{38}$, J.M. Carceller ${ }^{78}$, R. Caruso ${ }^{58,47}$, A. Castellina ${ }^{54,52}$, F. Catalani ${ }^{19}$, G. Cataldi ${ }^{48}$, L. Cazon ${ }^{72}$, M. Cerda ${ }^{10}$, J.A. Chinellato ${ }^{22}$, J. Chudoba ${ }^{32}$, L. Chytka ${ }^{33}$, R.W. Clay ${ }^{13}$, A.C. Cobos Ceruttii ${ }^{7}$, R. Colalillo ${ }^{60,50}$, A. Coleman ${ }^{93}$, M.R. Coluccia ${ }^{48}$, R. Conceição ${ }^{72}$, A. Condorelli ${ }^{45,46}$, G. Consolati ${ }^{49,55}$, F. Contreras ${ }^{11}$, F. Convenga ${ }^{56,48}$, D. Correia dos Santos $^{28}$, C.E. Covault ${ }^{85}$, S. Dasso ${ }^{5,3}$, K. Daumiller ${ }^{41}$, B.R. Dawson ${ }^{13}$, J.A. Day ${ }^{13}$, R.M. de Almeida $^{28}$, J. de Jesús ${ }^{8,41}$, S.J. de Jong ${ }^{80,81}$, G. De Mauro ${ }^{80,81}$, J.R.T. de Mello Neto ${ }^{26,27}$, I. De Mitri ${ }^{45,46}$, J. de Oliveira ${ }^{18}$, D. de Oliveira Franco ${ }^{22}$, F. de Palma ${ }^{56,48}$, V. de Souza $^{20}$, E. De Vito ${ }^{56,48}$, M. del Río ${ }^{11}$, O. Deligny ${ }^{34}$, L. Deval ${ }^{41,8}$, A. di Matteo $^{52}$, C. Dobrigkeit ${ }^{22}$, J.C. D’Olivo ${ }^{68}$, L.M. Domingues Mendes ${ }^{72}$, R.C. dos Anjos ${ }^{25}$, D. dos Santos $^{28}$, M.T. Dova ${ }^{4}$, J. Ebr ${ }^{32}$, R. Engel ${ }^{39,41}$, I. Epicoco ${ }^{56,48}$, M. Erdmann ${ }^{42}$, C.O. Escobar ${ }^{a}$, A. Etchegoyen ${ }^{8,12}$, H. Falcke ${ }^{80,82,81}$, J. Farmer ${ }^{92}$, G. Farrar ${ }^{90}$, A.C. Fauth ${ }^{22}$, N. Fazzini ${ }^{a}$, F. Feldbusch ${ }^{40}$, F. Fenu ${ }^{54,52}$,

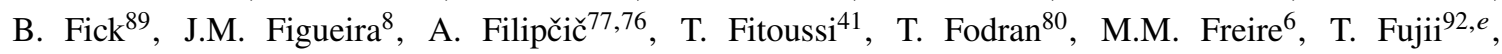
A. Fuster $^{8,12}$, C. Galea ${ }^{80}$, C. Galelli ${ }^{59,49}$, B. García ${ }^{7}$, A.L. Garcia Vegas ${ }^{42}$, H. Gemmeke ${ }^{40}$, F. Gesualdi ${ }^{8,41}$, A. Gherghel-Lascu ${ }^{73}$, P.L. Ghia ${ }^{34}$, U. Giaccari ${ }^{80}$, M. Giammarchi ${ }^{49}$, J. Glombitza ${ }^{42}$, F. Gobbi ${ }^{10}$, F. Gollan ${ }^{8}$, G. Golup ${ }^{1}$, M. Gómez Berisso ${ }^{1}$, P.F. Gómez Vitale ${ }^{11}$, J.P. Gongora ${ }^{11}$, J.M. González ${ }^{1}$, N. González ${ }^{14}$, I. Goos ${ }^{1,41}$, D. Góra ${ }^{70}$, A. Gorgi ${ }^{54,52}$, M. Gottowik ${ }^{38}$, T.D. Grubb ${ }^{13}$, F. Guarino ${ }^{60,50}$, G.P. Guedes ${ }^{23}$, E. Guido ${ }^{52,63}$, S. $\mathrm{Hahn}^{41,8}$, P. $\mathrm{Hamal}^{32}$, M.R. Hampel ${ }^{8}$, P. Hansen ${ }^{4}$, D. Harari ${ }^{1}$, V.M. Harvey ${ }^{13}$, A. Haungs ${ }^{41}$, T. Hebbeker ${ }^{42}$, D. Heck ${ }^{41}$, G.C. Hill ${ }^{13}$, C. Hojvat ${ }^{a}$, J.R. Hörandel ${ }^{80,81}$, P. Horvath ${ }^{33}$, M. Hrabovský ${ }^{33}$, T. Huege ${ }^{41,15}$, A. Insolia ${ }^{58,47}$, P.G. Isar $^{74}$, P. Janecek ${ }^{32}$, J.A. Johnsen ${ }^{86}$, J. Jurysek ${ }^{32}$, A. Kääpä ${ }^{38}$, K.H. Kampert ${ }^{38}$, N. Karastathis ${ }^{41}$, B. Keilhauer ${ }^{41}$, J. Kemp ${ }^{42}$, A. Khakurdikar ${ }^{80}$, V.V. Kizakke Covilakam $^{8,41}$, H.O. Klages ${ }^{41}$, M. Kleifges ${ }^{40}$, J. Kleinfeller ${ }^{10}$, M. Köpke ${ }^{39}$, N. Kunka ${ }^{40}$, B.L. Lago ${ }^{17}$, R.G. Lang ${ }^{20}$, N. Langner ${ }^{42}$, M.A. Leigui de Oliveira ${ }^{24}$, V. Lenok $^{41}$, A. Letessier-Selvon ${ }^{35}$, I. LhenryYvon $^{34}$, D. Lo Presti ${ }^{58,47}$, L. Lopes ${ }^{72}$, R. López ${ }^{64}$, L. Lu ${ }^{94}$, Q. Luce ${ }^{39}$, J.P. Lundquist ${ }^{76}$, A. Machado Payeras $^{22}$, G. Mancarella ${ }^{56,48}$, D. Mandat ${ }^{32}$, B.C. Manning ${ }^{13}$, J. Manshanden ${ }^{43}$, P. Mantsch ${ }^{a}$, S. Marafico ${ }^{34}$, A.G. Mariazzi ${ }^{4}$, I.C. Mariş ${ }^{14}$, G. Marsella ${ }^{61,47}$, D. Martello ${ }^{56,48}$, S. Martinelli ${ }^{41,8}$, O. Martínez Bravo ${ }^{64}$, M. Mastrodicasa ${ }^{57,46}$, H.J. Mathes ${ }^{41}$, J. Matthews ${ }^{88}$, G. Matthiae ${ }^{62,51}$, E. Mayotte ${ }^{38}$, P.O. Mazur ${ }^{a}$, G. MedinaTanco $^{68}$, D. Melo $^{8}$, A. Menshikov ${ }^{40}$, K.-D. Merenda ${ }^{86}$, S. Michal ${ }^{33}$, M.I. Micheletti ${ }^{6}$, L. Miramonti ${ }^{59,49}$, S. Mollerach ${ }^{1}$, F. Montanet ${ }^{36}$, C. Morello ${ }^{54,52}$, M. Mostafá ${ }^{91}$, A.L. Müller ${ }^{8}$, M.A. Muller ${ }^{22}$, K. Mulrey ${ }^{15}$, R. Mussa ${ }^{52}$, M. Muzio ${ }^{90}$, W.M. Namasaka ${ }^{38}$, A. Nasr-Esfahani ${ }^{38}$, L. Nellen ${ }^{68}$, M. Niculescu-Oglinzanu ${ }^{73}$, M. Niechciol ${ }^{44}$, D. Nitz ${ }^{89}$, D. Nosek ${ }^{31}$, V. Novotny ${ }^{31}$, L. Nožka ${ }^{33}$, A Nucita ${ }^{56,48}$, L.A. Núñez ${ }^{30}$, M. Palatka ${ }^{32}$, J. Pallotta ${ }^{2}$, P. Papenbreer ${ }^{38}$, G. Parente ${ }^{79}$, A. Parra ${ }^{64}$, J. Pawlowsky ${ }^{38}$, M. Pech ${ }^{32}$, F. Pedreira ${ }^{79}$, J. Pȩkala ${ }^{70}$, R. Pelayo ${ }^{65}$, J. Peña-Rodriguez ${ }^{30}$, E.E. Pereira Martins ${ }^{39,8}$, J. Perez Armand ${ }^{21}$, C. Pérez Bertolli $^{8,41}$, M. Perlin ${ }^{8,41}$, L. Perrone ${ }^{56,48}$, S. Petrera ${ }^{45,46}$, T. Pierog ${ }^{41}$, M. Pimenta ${ }^{72}$, V. Pirronello ${ }^{58,47}$, M. Platino ${ }^{8}$, B. Pont $^{80}$, M. Pothast ${ }^{81,80}$, P. Privitera ${ }^{92}$, M. Prouza ${ }^{32}$, A. Puyleart ${ }^{89}$, S. Querchfeld ${ }^{38}$, J. Rautenberg ${ }^{38}$, D. Ravignani ${ }^{8}$, M. Reininghaus ${ }^{41,8}$, J. Ridky ${ }^{32}$, F. Riehn ${ }^{72}$, M. Risse ${ }^{44}$, V. Rizi ${ }^{57,46}$, W. Rodrigues de Carvalho ${ }^{21}$, J. Rodriguez Rojo ${ }^{11}$, M.J. Roncoroni ${ }^{8}$, S. Rossoni ${ }^{43}$, M. Roth ${ }^{41}$, E. Roulet ${ }^{1}$, A.C. Rovero ${ }^{5}$, P. Ruehl ${ }^{44}$, A. Saftoiu ${ }^{73}$, F. Salamida ${ }^{57,46}$, H. Salazar ${ }^{64}$, G. Salina ${ }^{51}$, J.D. Sanabria Gomez ${ }^{30}$, F. Sánchez ${ }^{8}$, E.M. Santos ${ }^{21}$, E. Santos ${ }^{32}$, F. Sarazin ${ }^{86}$, R. Sarmento ${ }^{72}$, C. Sarmiento-Cano ${ }^{8}$, R. Sato ${ }^{11}$, 
P. Savina ${ }^{56,48,34,94}$, C.M. Schäfer ${ }^{41}$, V. Scherini ${ }^{56,48}$, H. Schieler ${ }^{41}$, M. Schimassek ${ }^{39,8}$, M. Schimp ${ }^{38}$, F. Schlüter ${ }^{41,8}$, D. Schmidt ${ }^{39}$, O. Scholten ${ }^{84,15}$, P. Schovánek ${ }^{32}$, F.G. Schröder ${ }^{93,41}$, S. Schröder ${ }^{38}$, J. Schulte ${ }^{42}$, S.J. Sciutto ${ }^{4}$, M. Scornavacche ${ }^{8,41}$, A. Segreto ${ }^{53,47}$, S. Sehgal ${ }^{38}$, R.C. Shellard ${ }^{16}$, G. Sigl ${ }^{43}$, G. Silli ${ }^{8,41}$, O. Sima ${ }^{73, f}$, R. Šmída ${ }^{92}$, P. Sommers ${ }^{91}$, J.F. Soriano ${ }^{87}$, J. Souchard ${ }^{36}$, R. Squartini ${ }^{10}$, M. Stadelmaier ${ }^{41,8}$, D. Stanca ${ }^{73}$, S. Stanič ${ }^{76}$, J. Stasielak ${ }^{70}$, P. Stassi ${ }^{36}$, A. Streich ${ }^{39,8}$, M. Suárez-Durán ${ }^{14}$, T. Sudholz ${ }^{13}$, T. Suomijärvi ${ }^{37}$, A.D. Supanitsky ${ }^{8}$, Z. Szadkowski ${ }^{71}$, A. Tapia ${ }^{29}$, C. Taricco ${ }^{63,52}$, C. Timmermans ${ }^{81,80}$, O. Tkachenko ${ }^{41}$, P. Tobiska ${ }^{32}$, C.J. Todero Peixoto ${ }^{19}$, B. Tomé ${ }^{72}$, Z. Torrès ${ }^{36}$, A. Travaini ${ }^{10}$, P. Travnicek $^{32}$, C. Trimarelli ${ }^{57,46}$, M. Tueros ${ }^{4}$, R. Ulrich ${ }^{41}$, M. Unger ${ }^{41}$, L. Vaclavek ${ }^{33}$, M. Vacula ${ }^{33}$, J.F. Valdés Galicia ${ }^{68}$, L. Valore ${ }^{60,50}$, E. Varela ${ }^{64}$, A. Vásquez-Ramírez ${ }^{30}$, D. Veberič ${ }^{41}$, C. Ventura ${ }^{27}$, I.D. Vergara Quispe ${ }^{4}$, V. Verzi ${ }^{51}$, J. Vicha ${ }^{32}$, J. Vink $^{83}$, S. Vorobiov ${ }^{76}$, H. Wahlberg ${ }^{4}$, C. Watanabe ${ }^{26}$, A.A. Watson ${ }^{c}$, M. Weber ${ }^{40}$, A. Weindl ${ }^{41}$, L. Wiencke ${ }^{86}$, H. Wilczyński ${ }^{70}$, M. Wirtz ${ }^{42}$, D. Wittkowski ${ }^{38}$, B. Wundheiler ${ }^{8}$, A. Yushkov $^{32}$, O. Zapparrata ${ }^{14}$, E. Zas ${ }^{79}$, D. Zavrtanik ${ }^{76,77}$, M. Zavrtanik ${ }^{77,76}$, L. Zehrer ${ }^{76}$

${ }^{1}$ Centro Atómico Bariloche and Instituto Balseiro (CNEA-UNCuyo-CONICET), San Carlos de Bariloche, Argentina

${ }^{2}$ Centro de Investigaciones en Láseres y Aplicaciones, CITEDEF and CONICET, Villa Martelli, Argentina

${ }^{3}$ Departamento de Física and Departamento de Ciencias de la Atmósfera y los Océanos, FCEyN, Universidad de Buenos Aires and CONICET, Buenos Aires, Argentina

${ }^{4}$ IFLP, Universidad Nacional de La Plata and CONICET, La Plata, Argentina

${ }^{5}$ Instituto de Astronomía y Física del Espacio (IAFE, CONICET-UBA), Buenos Aires, Argentina

${ }^{6}$ Instituto de Física de Rosario (IFIR) - CONICET/U.N.R. and Facultad de Ciencias Bioquímicas y Farmacéuticas U.N.R., Rosario, Argentina

${ }^{7}$ Instituto de Tecnologías en Detección y Astropartículas (CNEA, CONICET, UNSAM), and Universidad Tecnológica Nacional - Facultad Regional Mendoza (CONICET/CNEA), Mendoza, Argentina

${ }^{8}$ Instituto de Tecnologías en Detección y Astropartículas (CNEA, CONICET, UNSAM), Buenos Aires, Argentina

${ }^{9}$ International Center of Advanced Studies and Instituto de Ciencias Físicas, ECyT-UNSAM and CONICET, Campus Miguelete - San Martín, Buenos Aires, Argentina

${ }^{10}$ Observatorio Pierre Auger, Malargüe, Argentina

${ }^{11}$ Observatorio Pierre Auger and Comisión Nacional de Energía Atómica, Malargüe, Argentina

12 Universidad Tecnológica Nacional - Facultad Regional Buenos Aires, Buenos Aires, Argentina

${ }^{13}$ University of Adelaide, Adelaide, S.A., Australia

${ }^{14}$ Université Libre de Bruxelles (ULB), Brussels, Belgium

15 Vrije Universiteit Brussels, Brussels, Belgium

${ }^{16}$ Centro Brasileiro de Pesquisas Fisicas, Rio de Janeiro, RJ, Brazil

${ }^{17}$ Centro Federal de Educação Tecnológica Celso Suckow da Fonseca, Nova Friburgo, Brazil

${ }^{18}$ Instituto Federal de Educação, Ciência e Tecnologia do Rio de Janeiro (IFRJ), Brazil

${ }^{19}$ Universidade de São Paulo, Escola de Engenharia de Lorena, Lorena, SP, Brazil

${ }^{20}$ Universidade de São Paulo, Instituto de Física de São Carlos, São Carlos, SP, Brazil

${ }^{21}$ Universidade de São Paulo, Instituto de Física, São Paulo, SP, Brazil

${ }^{22}$ Universidade Estadual de Campinas, IFGW, Campinas, SP, Brazil

${ }^{23}$ Universidade Estadual de Feira de Santana, Feira de Santana, Brazil

${ }^{24}$ Universidade Federal do ABC, Santo André, SP, Brazil

${ }^{25}$ Universidade Federal do Paraná, Setor Palotina, Palotina, Brazil

${ }^{26}$ Universidade Federal do Rio de Janeiro, Instituto de Física, Rio de Janeiro, RJ, Brazil

${ }^{27}$ Universidade Federal do Rio de Janeiro (UFRJ), Observatório do Valongo, Rio de Janeiro, RJ, Brazil

${ }^{28}$ Universidade Federal Fluminense, EEIMVR, Volta Redonda, RJ, Brazil

${ }^{29}$ Universidad de Medellín, Medellín, Colombia

${ }^{30}$ Universidad Industrial de Santander, Bucaramanga, Colombia

${ }^{31}$ Charles University, Faculty of Mathematics and Physics, Institute of Particle and Nuclear Physics, Prague, Czech Republic

32 Institute of Physics of the Czech Academy of Sciences, Prague, Czech Republic 
${ }^{33}$ Palacky University, RCPTM, Olomouc, Czech Republic

34 CNRS/IN2P3, IJCLab, Université Paris-Saclay, Orsay, France

${ }^{35}$ Laboratoire de Physique Nucléaire et de Hautes Energies (LPNHE), Sorbonne Université, Université de Paris, CNRSIN2P3, Paris, France

${ }^{36}$ Univ. Grenoble Alpes, CNRS, Grenoble Institute of Engineering Univ. Grenoble Alpes, LPSC-IN2P3, 38000 Grenoble, France

${ }^{37}$ Université Paris-Saclay, CNRS/IN2P3, IJCLab, Orsay, France

38 Bergische Universität Wuppertal, Department of Physics, Wuppertal, Germany

${ }^{39}$ Karlsruhe Institute of Technology (KIT), Institute for Experimental Particle Physics, Karlsruhe, Germany

${ }^{40}$ Karlsruhe Institute of Technology (KIT), Institut für Prozessdatenverarbeitung und Elektronik, Karlsruhe, Germany

${ }^{41}$ Karlsruhe Institute of Technology (KIT), Institute for Astroparticle Physics, Karlsruhe, Germany

${ }^{42}$ RWTH Aachen University, III. Physikalisches Institut A, Aachen, Germany

${ }^{43}$ Universität Hamburg, II. Institut für Theoretische Physik, Hamburg, Germany

${ }^{44}$ Universität Siegen, Department Physik - Experimentelle Teilchenphysik, Siegen, Germany

${ }^{45}$ Gran Sasso Science Institute, L'Aquila, Italy

46 INFN Laboratori Nazionali del Gran Sasso, Assergi (L'Aquila), Italy

${ }^{47}$ INFN, Sezione di Catania, Catania, Italy

${ }^{48}$ INFN, Sezione di Lecce, Lecce, Italy

${ }^{49}$ INFN, Sezione di Milano, Milano, Italy

${ }^{50}$ INFN, Sezione di Napoli, Napoli, Italy

${ }^{51}$ INFN, Sezione di Roma "Tor Vergata", Roma, Italy

52 INFN, Sezione di Torino, Torino, Italy

53 Istituto di Astrofisica Spaziale e Fisica Cosmica di Palermo (INAF), Palermo, Italy

54 Osservatorio Astrofisico di Torino (INAF), Torino, Italy

55 Politecnico di Milano, Dipartimento di Scienze e Tecnologie Aerospaziali , Milano, Italy

${ }^{56}$ Università del Salento, Dipartimento di Matematica e Fisica "E. De Giorgi”, Lecce, Italy

57 Università dell'Aquila, Dipartimento di Scienze Fisiche e Chimiche, L'Aquila, Italy

58 Università di Catania, Dipartimento di Fisica e Astronomia, Catania, Italy

${ }^{59}$ Università di Milano, Dipartimento di Fisica, Milano, Italy

${ }^{60}$ Università di Napoli "Federico II", Dipartimento di Fisica "Ettore Pancini”, Napoli, Italy

${ }^{61}$ Università di Palermo, Dipartimento di Fisica e Chimica "E. Segrè", Palermo, Italy

62 Università di Roma "Tor Vergata", Dipartimento di Fisica, Roma, Italy

${ }^{63}$ Università Torino, Dipartimento di Fisica, Torino, Italy

${ }^{64}$ Benemérita Universidad Autónoma de Puebla, Puebla, México

${ }^{65}$ Unidad Profesional Interdisciplinaria en Ingeniería y Tecnologías Avanzadas del Instituto Politécnico Nacional (UPIITA-IPN), México, D.F., México

66 Universidad Autónoma de Chiapas, Tuxtla Gutiérrez, Chiapas, México

${ }^{67}$ Universidad Michoacana de San Nicolás de Hidalgo, Morelia, Michoacán, México

${ }^{68}$ Universidad Nacional Autónoma de México, México, D.F., México

${ }^{69}$ Universidad Nacional de San Agustin de Arequipa, Facultad de Ciencias Naturales y Formales, Arequipa, Peru

${ }^{70}$ Institute of Nuclear Physics PAN, Krakow, Poland

${ }^{71}$ University of Łódź, Faculty of High-Energy Astrophysics, Łódź, Poland

${ }^{72}$ Laboratório de Instrumentação e Física Experimental de Partículas - LIP and Instituto Superior Técnico - IST, Universidade de Lisboa - UL, Lisboa, Portugal

73 "Horia Hulubei” National Institute for Physics and Nuclear Engineering, Bucharest-Magurele, Romania

${ }^{74}$ Institute of Space Science, Bucharest-Magurele, Romania

75 University Politehnica of Bucharest, Bucharest, Romania

76 Center for Astrophysics and Cosmology (CAC), University of Nova Gorica, Nova Gorica, Slovenia

${ }^{77}$ Experimental Particle Physics Department, J. Stefan Institute, Ljubljana, Slovenia

78 Universidad de Granada and C.A.F.P.E., Granada, Spain

${ }^{79}$ Instituto Galego de Física de Altas Enerxías (IGFAE), Universidade de Santiago de Compostela, Santiago de Compostela, Spain 
${ }^{80}$ IMAPP, Radboud University Nijmegen, Nijmegen, The Netherlands

${ }^{81}$ Nationaal Instituut voor Kernfysica en Hoge Energie Fysica (NIKHEF), Science Park, Amsterdam, The Netherlands

82 Stichting Astronomisch Onderzoek in Nederland (ASTRON), Dwingeloo, The Netherlands

${ }^{83}$ Universiteit van Amsterdam, Faculty of Science, Amsterdam, The Netherlands

${ }^{84}$ University of Groningen, Kapteyn Astronomical Institute, Groningen, The Netherlands

85 Case Western Reserve University, Cleveland, OH, USA

86 Colorado School of Mines, Golden, CO, USA

${ }^{87}$ Department of Physics and Astronomy, Lehman College, City University of New York, Bronx, NY, USA

${ }^{88}$ Louisiana State University, Baton Rouge, LA, USA

${ }^{89}$ Michigan Technological University, Houghton, MI, USA

${ }^{90}$ New York University, New York, NY, USA

${ }^{91}$ Pennsylvania State University, University Park, PA, USA

92 University of Chicago, Enrico Fermi Institute, Chicago, IL, USA

93 University of Delaware, Department of Physics and Astronomy, Bartol Research Institute, Newark, DE, USA

94 University of Wisconsin-Madison, Department of Physics and WIPAC, Madison, WI, USA

${ }^{a}$ Fermi National Accelerator Laboratory, Fermilab, Batavia, IL, USA

${ }^{b}$ Max-Planck-Institut für Radioastronomie, Bonn, Germany

${ }^{c}$ School of Physics and Astronomy, University of Leeds, Leeds, United Kingdom

${ }^{d}$ Colorado State University, Fort Collins, CO, USA

$e^{e}$ now at Hakubi Center for Advanced Research and Graduate School of Science, Kyoto University, Kyoto, Japan

$f$ also at University of Bucharest, Physics Department, Bucharest, Romania 